\title{
Goethes Einquartierungen Zur autobiographischen Dimensionalität besetzter Räume
}

MARTINA WAGNER-EGELHAAF

Da das wissenschaftliche Paradigma der ,Biographie' hermeneutisch im Historismus des 19. Jahrhunderts fundiert ist, hat die Autobiographieforschung in der Vergangenheit eher zeitliche als räumliche Untersuchungsperspektiven verfolgt. Die konstitutive Verbindung der Autobiographie mit der Dimension der Zeit liegt insofern auf der Hand, als sich das autobiographische Ich über den Akt der Erinnerung in die Vergangenheit zurückversetzt und damit eine zeitliche Differenz zwischen Erzählzeit und erzählter Zeit zu überwinden ist. ${ }^{1}$ Eine in der Autobiographieforschung grundlegende, obwohl immer wieder problematisierte Definition der Autobiographie geht auf den französischen Autobiographietheoretiker Philippe Lejeune zurück, der die Autobiographie bestimmt hat als ,[r]ückblickende Prosaerzählung einer tatsächlichen Person über ihre eigene Existenz, wenn sie den Nachdruck auf ihr persönliches Leben und insbesondere auf die Geschichte ihrer Persönlichkeit legt. “2 Der Autobiograph/die Autobiographin blickt also zurück in der Zeit, um eine Geschichte, d.h. eine Entwicklung zu erzählen, die den Zeitpunkt des Erzählens wieder einholt bzw. sich an ihn annähert. Dass dies im Ansatz ein hermeneutisches Unterfangen ist, wird unmissverständlich in einer Kapitelüberschrift von Christa Wolfs Kindheitsmuster deutlich, die eine Verstehensfrage stellt, nämlich: „Wie sind wir so geworden, wie wir heute sind?“3

Im Zuge des ,topographical' bzw. des ,spatial turn', die in den letzten Jahren eine quer durch die traditionellen Geistes- und Sozialwissenschaften zu verfolgende wissenschaftliche Aufmerksamkeit auf die räumliche Dimension kultureller Phänomene mit sich gebracht haben, ${ }^{4}$ fragt nun auch die Autobio- 
graphieforschung nach der Rolle von Räumen und Orten für die Konstitution des autobiographischen Ichs und in der Folge des autobiographischen Genres als solchem. ${ }^{5}$ Spätestens seit Kant gelten Raum und Zeit als die grundlegenden Anschauungsformen des Menschen, d.h. sie sind jeder Wahrnehmung und Begriffsbildung vorgeschaltet. In der Kritik der reinen Vernunft schreibt der Königsberger Philosoph, dass der Mensch mit dem inneren Sinn, also wenn er in sich hineinblickt, zeitlich denkt, aber sich mit dem äußeren Sinn die Gegenstände der Welt als im Raum befindlich vorstellt. ${ }^{6}$ Präferierte die Hermeneutik die Zeit als wissenschaftliche Kategorie, hat räumlich zu denken, in Anordnungen und Konstellationen, wissenschaftsgeschichtlich der Strukturalismus gelehrt, der sich indessen mit der Frage konfrontiert sieht, ob die Strukturen, die der Strukturalist erkennt, im Objekt anzutreffen sind oder im Kopf des Strukturalisten. ${ }^{7}$ Wie auch immer: Es scheint in der Autobiographie um beides gehen zu müssen, um Zeit und Raum, Außen und Innen, Subjekt und Objekt, und zwar in ihrer wechselseitigen Verschränkung, die für die Autobiographieforscherin im Text und nur dort zu verorten und zu untersuchen ist.

Allerdings befindet sich die literatur- und kulturwissenschaftliche Forschung längst in einer Phase nach der Hermeneutik, nach dem Strukturalismus und auch nach dem Poststrukturalismus, der, beispielsweise in Jacques Derridas différance-Begriff, Zeitlichkeit und Räumlichkeit zusammenzudenken versucht hat mit dem Ergebnis und dem Postulat einer unendlichen Differenzialität der Zeichenprozesse. ${ }^{8}$ Die Literaturwissenschaft hat heute die Lizenz, auf die verschiedenen Register zugreifen zu können, ohne sie absolut setzen oder gegeneinander ausspielen zu müssen, nach Maßgabe dessen, was ihr Erkenntnisinteresse an sehr konkreten literarischen Texten leitet. Autobiographie vom Raum her zu denken ermöglicht gegenüber der zeitorientierten geschichtlichen Perspektive eine andere Dimensionalität des autobiographischen Ichs. Da Raum und Zeit kategorial aufeinander bezogen sind, affiziert die räumliche Perspektive jedoch immer auch die Zeitwahrnehmung, die gleichsam spatialisiert wird. ${ }^{9}$

Indessen tut man gut daran, aus methodischen Gründen zwischen ,Raum“ und ,Ort ${ }^{`} \mathrm{zu}$ unterscheiden. ${ }^{10}$ Der ,Ort ${ }^{`}$ ist stets ein bestimmter Ort, der bezeichnet werden kann, gewissermaßen ein Punkt auf der Landkarte. Deswegen ist der Ort eine Kategorie, die sich systematisch eher dem ,topographical turn“ zuordnet, geht es hier doch um die Repräsentation (vgl. gr. , $\gamma \rho \alpha \varphi \varepsilon v v^{\prime}$, schreiben, malen, ritzen') von Orten (vgl. gr. , $\tau$ o $\pi \circ \varsigma^{`}$,Ort, Stelle, Gegend, Platz, Raum'). Der ,Raum', eine Kategorie des ,spatial turn“ (von lat. ,spatium“ 
,Strecke', ,Raum', ,Zeitraum', ,Frist'), ist eine Ausdehnung, eine Dimensionalität, man könnte auch sagen eine Erfahrung, in der sich ein wahrnehmendes Subjekt als positioniert erfährt. ${ }^{11}$ Ein Ort, so könnte man sagen, ist ein Punkt im Raum, aber jeder Ort eröffnet seinerseits die unterschiedlichsten Räume.

Die rhetorische Tradition, die den analytischen Blick auf die konkrete sprachliche Verfasstheit von mündlichen und schriftlichen Redeäußerungen richtet, liefert zwei Zugänge zur Räumlichkeit des Gedächtnisses, das als Vermögen jeglichem Akt der Erinnerung und damit in konstitutiver Weise der Autobiographie zugrunde liegt. Da ist zum einen die Memorialehre, die dem Redner empfiehlt, im vierten Produktionsstadium seiner Rede in den Räumen, den ,loci', einer imaginären Architektur bestimmte Bilder, ,imagines', abzulegen und sie beim Halten der Rede wieder einzusammeln. ${ }^{12}$ In diesem Sinne spricht der Kirchenvater Augustinus, dessen Confessiones (um 400) einen Markstein in der Geschichte der Autobiographie darstellen, von den „Gefilden und weiten Hallen des Gedächtnisses“ oder auch von „der weiten Behausung [s]eines Gedächtnisses““ ${ }^{13}$ Zum anderen ist die Topik zu nennen, deren zentrale Kategorie, der , $\tau \circ \pi \circ \varsigma^{\varsigma}$, ebenfalls räumlich gedacht ist. Aristoteles stellt einen direkten Bezug der mnemotechnischen Orte zu den Orten der topischen inventio her. ${ }^{14}$ Der rhetorische Ansatz ist für die literaturwissenschaftliche Analyse deshalb besonders fruchtbar, weil er die Sprachlichkeit dessen, was er beschreibt, profiliert und die Räumlichkeit des Texts auf diese Weise immer schon mit der Räumlichkeit des Referenzobjekts verschränkt. Insofern als die Topik mit ihren ,Gemeinplätzen' auf dem kulturellen Archiv des sensus communis basiert, konnte Stefan Goldmann eine autobiographische Topik herausarbeiten, die jeden autobiographischen Text, so Goldmann, in der Erzählung seiner scheinbar individuellen Lebensgeschichte kulturell vorgeprägte Orte abschreiten lässt. ${ }^{15}$ Ein solcher individueller und zugleich kultureller Ort ist in der Topologie der abendländischen Autobiographie zweifellos das Elternhaus, der erste (begrenzte) Lebensraum des Menschen, über den ihm die Welt vermittelt wird und von dem er gewissermaßen seinen ,Ausgang ${ }^{6}$ nimmt. ${ }^{16}$

Goethe, dessen unter dem Titel Aus meinem Leben. Dichtung und Wahrheit (1811-1833) veröffentlichte Autobiographie eine Schlüsselrolle in der germanistischen Autobiographieforschung innehat, weil sie eine prototypische Ausprägung des hermeneutischen bildungs- und entwicklungsgeschichtlichen $\mathrm{Au}-$ tobiographiemodells darstellt, beginnt das erste Kapitel seiner Lebensgeschichte gleichsam mit einem Paukenschlag, der Ort und Zeit punktartig zusammenführt: 
„Am 28. August 1749, Mittags mit dem Glockenschlage zwölf, kam ich in Frankfurt am Main auf die Welt. Die Konstellation war glücklich; die Sonne stand im Zeichen der Jungfrau, und kulminierte für den Tag; Jupiter und Venus blickten sie freundlich an, Merkur nicht widerwärtig; Saturn und Mars verhielten sich gleichgültig: nur der Mond, der so eben voll ward, übte die Kraft seines Gegenscheins um so mehr, als zugleich seine Planetenstunde eingetreten war. Er widersetzte sich daher meiner Geburt, die nicht eher erfolgen konnte, als bis diese Stunde vorübergegangen. “17

Mit dem Hinweis auf die Sternenkonstellation zum Zeitpunkt seiner Geburt zitiert Goethe einen traditionellen lebensgeschichtlichen Topos, der das Schicksal des Menschen in den Sternen geschrieben sieht und den er in Geronimo Cardanos De propria vita (1643) vorgeprägt fand. ${ }^{18}$ Im Anschluss daran eröffnet der autobiographische Erzähler seinen Leserinnen und Lesern den Raum bzw. die Räume des Frankfurter Elternhauses. Bezeichnenderweise verbindet er die „Hausführung“ mit einem Hinweis auf das Funktionieren der Erinnerung, wie es auch schon von Augustinus ${ }^{19}$ beschrieben ist:

\begin{abstract}
„Wenn man sich erinnern will, was uns in der frühsten Zeit der Jugend begegnet ist, so kommt man oft in den Fall, dasjenige was wir von andern gehört, mit dem zu verwechseln, was wir wirklich aus eigner anschauender Erfahrung besitzen. Ohne also hierüber eine genaue Untersuchung anzustellen, welche ohnehin zu nichts führen kann, bin ich mir bewußt, daß wir in einem alten Hause wohnten, welches eigentlich aus zwei durchbrochnen Häusern bestand. Eine turmartige Treppe führte zu unzusammenhangenden Zimmern, und die Ungleichheit der Stockwerke war durch Stufen ausgeglichen. Für uns Kinder, eine jüngere Schwester und mich, war der untere weitläuftige Hausflur der liebste Raum, welcher neben der Türe ein großes hölzernes Gitterwerk hatte, wodurch man unmittelbar mit der Straße und der freien Luft in Verbindung kam. Einen solchen Vogelbauer, mit dem viele Häuser versehen waren, nannte man ein Geräms.“"(DW S. 15f.)
\end{abstract}

Es schließt sich die berühmte, von Freud interpretierte Szene an, in der der kleine Goethe Geschirr aus dem Geräms auf die Straße wirft. ${ }^{20}$ Auf sie kann hier nicht näher eingegangen werden, signifikant für die , anschauende Erinnerung' ist aber die Vorstellung des alten Hauses, das in der Vertikale den Eindruck des Verwinkelten und Unregelmäßigen hervorruft, in der Horizontale 
Raum und Weitläufigkeit und dazu Kontakt mit der Außenwelt und damit eine Außen-Innen-Korrespondenz zu denken gibt. ${ }^{21}$ Im Anschluss an den Hausflur befindet sich ein großes Zimmer, in dem die Großmutter väterlicherseits lebt und das die Kinder in ihre Spiele einbeziehen (vgl. DW S. 17).

Auf der Hinterseite des Hauses, so beschreibt Goethe weiter, eröffnet sich vom oberen Stock ein Blick ,über eine beinah unabsehbare Fläche von Nachbarsgärten“, allerdings verfügt das an einer Straßenecke gelegene Haus der Familie Goethe am Hirschgraben nicht über einen Garten, ist vielmehr ,durch eine ziemlich hohe Mauer [des] Hofes von diesen so nah gelegenen Paradiesen ausgeschlossen“ (DW S. 18). Diese architekturale Anlage, der Blick aus dem Haus in die unzugänglichen Gärten der Nachbarn, wird Goethe im Rückblick zur Matrix einer Selbstdeutung:

„Im zweiten Stock befand sich ein Zimmer, welches man das Gartenzimmer nannte, weil man sich daselbst durch wenige Gewächse vor dem Fenster den Mangel eines Gartens zu ersetzen gesucht hatte. Dort war, wie ich heranwuchs, mein liebster, zwar nicht trauriger, aber doch sehnsüchtiger Aufenthalt. Über jene Gärten hinaus, über Stadtmauern und Wälle sah man in eine schöne fruchtbare Ebene; es ist die, welche sich nach Höchst hinzieht. Dort lernte ich Sommerszeit gewöhnlich meine Lektionen, wartete die Gewitter ab, und konnte mich an der untergehenden Sonne, gegen welche die Fenster gerade gerichtet waren, nicht satt genug sehen. Da ich aber zu gleicher Zeit die Nachbarn in ihren Gärten wandeln und ihre Blumen besorgen, die Kinder spielen, die Gesellschaften sich ergetzen sah, die Kegelkugeln rollen und die Kegel fallen hörte; so erregte dies frühzeitig in mir ein Gefühl der Einsamkeit und einer daraus entspringenden Sehnsucht, das dem von der Natur in mich gelegten Ernsten und Ahnungsvollen entsprechend, seinen Einfluß gar bald und in der Folge noch deutlicher zeigte.“ (DW S. 18)

Das ,Ausgeschlossensein aus dem Paradies' stellt eine markante autobiographietheoretische Signatur dar, insofern als der Mensch erst durch den Verlust des Paradieses auf die irdische Lebensbahn geworfen und damit sterblich wird. Und es ist der Tod, der dem Leben erst seinen Sinn verleiht und auf diese Weise die autobiographische Rückbesinnung motiviert. Diese grundlegende Figur des menschlichen Daseins wird von Goethe in der zitierten Stelle zur individuellen Lebensszene gestaltet. Erzeugt der Gartenblick eine melancholischromantische Stimmung, vermag das Haus bemerkenswerterweise aber auch 


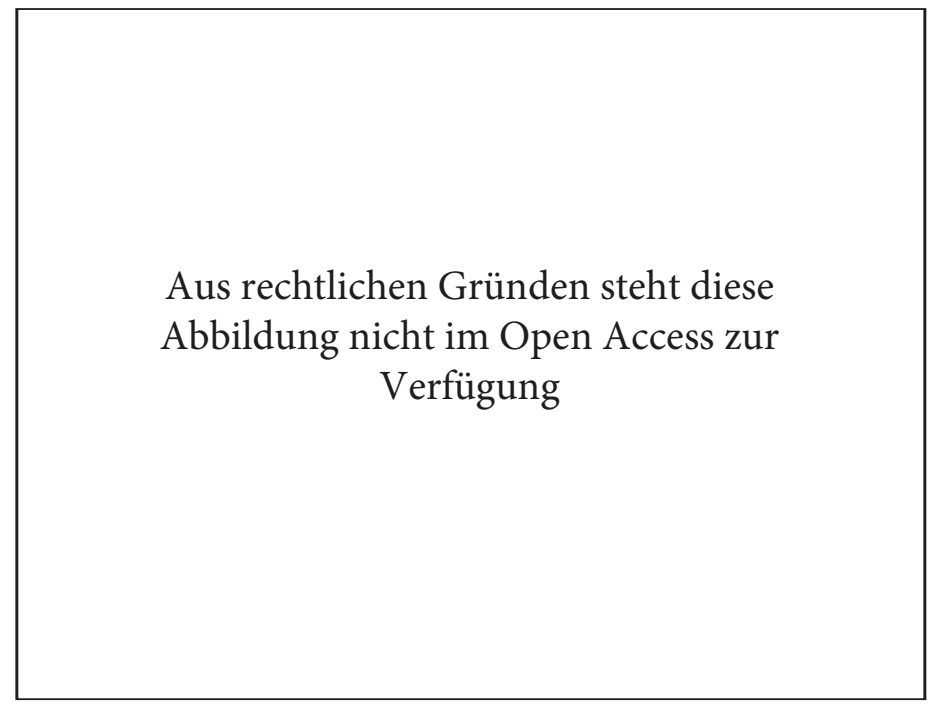

Abb. 1: Puppentheater. Frankfurt a. M., Goethe-Haus

das dramatische Register zu bedienen, das seit der Poetik des Aristoteles

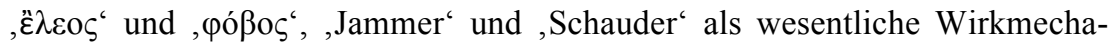
nismen der Tragödie ansetzt. ${ }^{22}$ Bei Goethe heißt es: „Die alte, winkelhafte, an vielen Stellen düstere Beschaffenheit des Hauses war übrigens geeignet, Schauer und Furcht in kindlichen Gemütern zu erwecken" (DW S. 18). Nur wenige Abschnitte später erfährt man, dass die Großmutter, ,in deren geräumigem Wohnzimmer“ die Kinder „hinlänglich Platz zu [ihren] Spielen fanden“ den Kindern „ein Puppenspiel vorstellen ließ“, eine „kleine Bühne“, die

\footnotetext{
„in dem alten Hause eine neue Welt erschuf. Dieses unerwartete Schauspiel zog die jungen Gemüter mit Gewalt an sich; besonders auf den Knaben machte es einen sehr starken Eindruck, der in eine große langandauernde Wirkung nachklang." (DW S. 20) (Abb. 1)
}

Hier, in einem abgegrenzten Raum innerhalb eines abgegrenzten Raums, der „in dem alten Hause eine neue Welt erschuf", übt sich also schon der spätere Dramatiker und misst die dramatischen Spielräume aus. Auch Goethes Italien- 


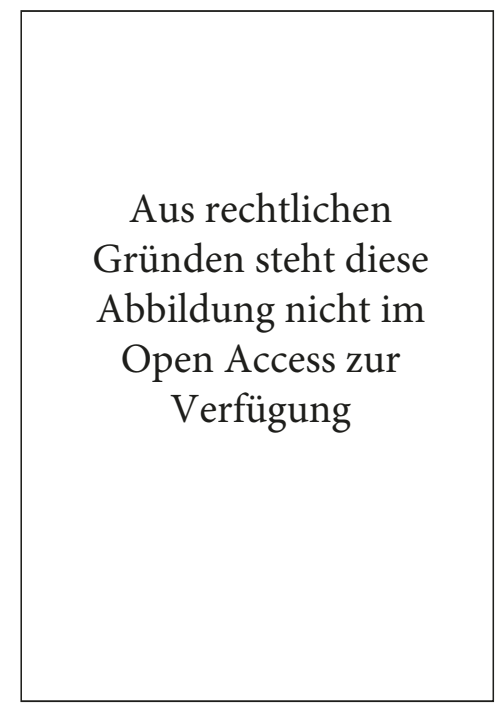

Abb. 2: Treppenhaus. Frankfurt a. M., Goethe-Haus

reise und die klassische Kunsterfahrung sind im Elternhaus bereits vorgebaut und letztere prägt, so stellt der Autobiograph es dar, den Geist des jungen Johann Wolfgang. Da heißt es:

„Innerhalb des Hauses zog meinen Blick am meisten eine Reihe römischer Prospekte auf sich, mit welchen der Vater einen Vorsaal ausgeschmückt hatte, gestochen von einigen geschickten Vorgängern des Piranese, die sich auf Architektur und Perspektive wohl verstanden, und deren Nadel sehr deutlich und schätzbar ist. Hier sah ich täglich die Piazza del Popolo, das Coliseo, den Petersplatz, die Peterskirche von außen und innen, die Engelsburg und so manches andere. Diese Gestalten drückten sich tief bei mir ein [...].“ (DW S. 19) (Abb. 2)

In der Enge des Hauses eröffnen sich also die weiten römischen Prospekte als Deutungsperspektive der Goethe'schen Autobiographie, deren Fortsetzung ja gerade die 1816, 1817 und 1829 veröffentlichte Italienische Reise bildet. Goethe berichtet des Weiteren, wie der Vater nach dem Tod der Großmutter be- 
gann, das Haus umzubauen, allerdings, um eine bestimmte Bauvorschrift zu umgehen, bei, heute würde man sagen „laufendem Betrieb“. Für die Kinder bedeutet dies eine gänzlich neue Raumerfahrung:

\begin{abstract}
„Die Zimmer, in denen man sie oft enge genug gehalten und mit wenig erfreulichem Lernen und Arbeiten geängstigt, die Gänge, auf denen sie gespielt, die Wände, für deren Reinlichkeit und Erhaltung man sonst so sehr gesorgt, alles das vor der Hacke des Maurers, vor dem Beile des Zimmermanns fallen zu sehen, und zwar von unten herauf, und indessen oben auf unterstützten Balken, gleichsam in der Luft zu schweben, und dabei immer noch zu einer gewissen Lektion, zu einer bestimmten Arbeit angehalten zu werden - dieses alles brachte eine Verwirrung in den jungen Köpfen hervor, die sich so leicht nicht wieder ins Gleiche setzen ließ. Doch wurde die Unbequemlichkeit von der Jugend weniger empfunden, weil ihr etwas mehr Spielraum als bisher, und manche Gelegenheit, sich auf Balken zu schaukeln und auf Brettern zu schwingen, gelassen ward." (DW S. 21f.)
\end{abstract}

Für den jungen Goethe öffnet sich mit diesem Umbau des Elternhauses der Blick nach draußen. Der begrenzte Kosmos des elterlichen Hauses erweitert sich und entsprechend fährt die Autobiographie mit der Schilderung der äußeren Umgebung und des Stadtraums fort, den das selbständiger werdende autobiographische Ich zunehmend erkundet. Allerdings spielt das Elternhaus in Goethes Lebensbeschreibung an späterer Stelle nochmals eine bedeutsame Rolle: Im dritten Buch von Dichtung und Wahrheit schildert der autobiographische Erzähler sehr ausführlich, wie die Familie Goethe im Zuge des Siebenjährigen Kriegs, der die Franzosen nach Frankfurt brachte, französische Einquartierung hinnehmen muss. Ins Haus kommt der französische Königsleutnant Graf Thorane, ein rücksichtsvoller, zivilisierter, kunstsinniger, höflicher und äußerst tüchtiger Militär, dessen Aufgabe darin bestand, Zivilstreitigkeiten zwischen Soldaten und Bürgern zu schlichten. Er bleibt zwei Jahre im Haus und ist so rücksichtsvoll, dass er noch nicht einmal seine Landkarten an die Wände nagelt, um die neuen Goethe'schen Tapeten zu schonen. Die Familie muss dem Grafen und seinen Leuten ihre ,wohlaufgeputzten und meist verschlossenen Staatszimmer einräumen“ (DW S. 94) (Abb. 3) und sich selbst in andere Räume des Hauses zurückziehen.

Der Graf bemüht sich um ein gutes, ja freundschaftliches Auskommen mit dem Hausherrn und seiner Familie, trifft aber auf den geballten Groll von Goe- 


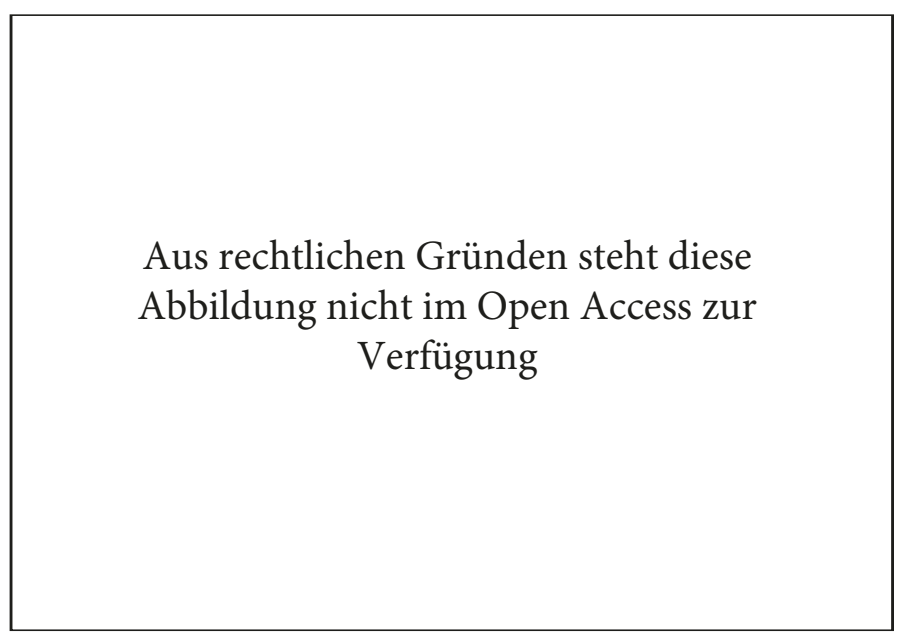

Abb. 3: Peking-Zimmer. Frankfurt a. M., Goethe-Haus

thes Vater, während es ihm durchaus gelingt, die Mutter und die Kinder für sich einzunehmen. Die Anwesenheit des Fremden in seinem Haus ist Goethes Vater ein unüberwindliches Ärgernis; Mutter und Kindern jedoch ist klar, dass alles viel schlimmer hätte kommen können, d.h. dass weniger zivilisierte und rücksichtsvolle Soldaten im Goethe'schen Haus hätten einquartiert werden können. Die Mutter lernt eifrig französisch, die Kinder profitieren von den feinen Desserts der französischen Tafel und der junge Goethe findet die Begegnungen mit den Frankfurter Künstlern, die der Graf ins Haus holt, um sich von ihnen Tapeten für das Schloss seines Bruders in der Provence malen zu lassen, mehr als anregend. Dass Graf Thorane Frankfurter Künstler für die Tapeten seines Bruders anstellt, spricht nicht nur für sein Kunstinteresse, sondern auch dafür, dass er den Wert, den ein wohlausgestattetes Haus für seine Besitzer und Bewohner hat, zu schätzen weiß und sich auch deswegen so rücksichtsvoll im Hause der Familie Goethe verhält. Dies stimmt Johann Caspar Goethe allerdings keineswegs gewogener, er beharrt vielmehr darauf, dass er der Herr des Hauses ist und in seinem Haus der französische Graf nichts verloren hat. Eines Tages kommt es zu einem Eklat, bezeichnenderweise auf der Treppe des Hauses (Abb. 2), auf die Goethe in Dichtung und Wahrheit mehrfach zu sprechen kommt (vgl. DW S. 15/95). Der Treppe, die Goethes Vater besonders re- 
präsentativ hatte gestalten lassen - sie enthält sogar das Monogramm des Hausherrn und seiner Frau ${ }^{23}$-, kommt im Haus eine zentrale Bedeutung zu, verbindet sie doch die Räume des Hauses miteinander und stellt auf diese Weise die nicht vorhandene Einheit des verwinkelten Hauses her. Nach dem Sieg der Franzosen über die Hannover'sche Armee unter Herzog Ferdinand von Braunschweig bei Bergen (zwischen Frankfurt und Hanau) am 13. April 1759 ereignet sich Folgendes im Hause Goethe:

\begin{abstract}
„Die Treppe lief frei durchs ganze Haus an allen Vorsälen vorbei. Der Vater mußte, indem er herabstieg, unmittelbar an des Grafen Zimmer vorübergehn. Sein Vorsaal stand so voller Leute, daß der Graf sich entschloß, um mehrers auf Einmal abzutun, herauszutreten; und dies geschah leider in dem Augenblick als der Vater herabkam. Der Graf ging ihm heiter entgegen, begrüßte ihn und sagte ,Ihr werdet uns und Euch Glück wünschen, daß diese gefährliche Sache so glücklich abgelaufen ist.' - ,Keineswegs!' versetzte mein Vater, mit Ingrimm; ,ich wollte sie hätten Euch zum Teufel gejagt, und wenn ich hätte mitfahren sollen.“"(DW S. 112)
\end{abstract}

Die Konstellation ist sprechend: Vater Goethe schreitet die herrschaftliche Treppe in seinem Haus herab - im Code des Absolutismus eine Geste der Herablassung seitens eines Souveräns, wenn er Gäste begrüßt - und dann trifft er auf den ungeladenen und missliebigen ,Gast', der aus seinem (Johann Caspar Goethes) repräsentativen, aber zweckentfremdeten Wohnzimmer tritt und unmissverständlich zu verstehen gibt, wer der wahre Souverän im Haus ist. $\mathrm{Ob}$ der unbotmäßigen Antwort des depotenzierten Hausherrn verliert nun auch der sonst so beherrschte Thorane die Geduld, wird seinerseits wütend und, um klar und deutlich zu zeigen, bei wem die Herrschaftsgewalt liegt, veranlasst er, dass der störrische Johann Caspar Goethe auf die Wache abgeführt wird. In seinem eigenen Haus festgenommen und abgeführt zu werden, ist freilich für einen ,Hausherrn“ mehr als ehrenrührig. Allerdings lässt der Graf mit sich verhandeln: einem befreundeten Dolmetsch gelingt es, ihn nach vielem Zureden dazu zu bringen, sich zu überwinden und Vater Goethe wieder auf freien Fuß zu setzen - der indessen feindselig wie zuvor bleibt und alles daran setzt, den französischen Eindringling aus seinem Hause zu entfernen, was ihm nach annähernd zwei Jahren schließlich auch gelingt. Damit ist die ,wahre Hausherrschaft' wieder hergestellt - Johann Caspar ist wieder, frei nach Freud, „Herr in seinem eigenen Haus“. ${ }^{24}$ 
Bemerkenswerterweise sind die Sympathien des jungen Goethe in dieser Konstellation auf Seiten des französischen Grafen. Klaus-Detlef Müller hat sogar davon gesprochen, dass der Königsleutnant Thorane für den jungen Goethe ,in dem Maße zur positiven Vaterfigur wird, wie der Rat Goethe sich unvernünftig in seiner Hypochondrie verrennt.“.25 Das Elternhaus stellt also gleichsam die theatrale Szene einer retrospektiven Selbstwahrnehmung und -deutung des autobiographischen Ichs dar, das einer starren Außen/Innen- und entsprechend einer Fremd/Eigen-Dichotomie entwächst und die identifikatorische Wahrnehmung des starken Charakters an dessen civilité und die Fähigkeit des Verhandelns und der Selbstüberwindung knüpft. Dabei ist es die architekturale Anlage, die dem Setting über die Markierung und Abgrenzung von Räumen einen interpretatorischen Rahmen verleiht.

Der Frankfurter Einquartierungsszene lässt sich der Eintrag vom 4. Oktober (1792) aus Goethes Kriegstagebuch Campagne in Frankreich 1792 zur Seite stellen. Campagne in Frankreich und Belagerung von Mainz bilden den fünften Teil der zweiten Abteilung von Goethes Aus meinem Leben untertitelter Autobiographie. Sie wurden 1822 bei Cotta in Stuttgart und Tübingen gedruckt. Eher gegen seinen Willen muss Goethe, der sich in Weimar häuslich niedergelassen und eine Familie gegründet hatte, Herzog Carl August auf dem österreichisch-preußischen Feldzug gegen das revolutionäre Frankreich begleiten. Goethe reist mit einem Auszug des von Johann Wilhelm Abraham Jäger herausgegebenen topographischen Grand Atlas d'Allemagne en LXXXI feuilles von 1789 im Gepäck. ${ }^{26}$ Zwischen die Kriegserfahrung selbst und den Text der Campagne ist gleichsam ein abstrahierendes graphisches Medium geschaltet, das im Kriegskontext dem pragmatischen Zweck der Orientierung auf unbekanntem Terrain dient, retrospektiv aber zum autobiographischen Erinnerungsmedium wird:

„In Maynz hatte mich Herr v. Stein mit dem Jägerischen Atlas versorgt, welcher den gegenwärtigen, hoffentlich auch den nächstkünftigen Kriegsschauplatz in mehreren Blättern darstellte: Ich nahm das eine hervor, das achtundvierzigste, in dessen Bezirk ich bei Longwy hereingetreten war, und da unter des Herzogs Leuten sich gerade ein Boßler befand, so ward es zerschnitten und aufgezogen und dient mir noch zur Wiedererinnerung jener für die Welt und mich so bedeutenden Tage.“ (C S. 403; vgl. C S. 410) 
Am 4. Oktober befindet man sich bereits auf dem Rückzug nach der Kanonade von Valmy, deren wenig rühmlichen Ausgang Goethe in autobiographischer Selbstgewissheit bekanntlich mit den Worten zusammenfasste: „von hier und heute geht eine neue Epoche der Weltgeschichte aus, und ihr könnt sagen, ihr seid dabei gewesen“ (C S. 436). Ort (,hier“) und Zeit (,heute“) werden in einer souveränen Geste der Welt- und Selbstdeutung zur „Epoche“, ja gar zur „neue[n] Epoche der Weltgeschichte“ ${ }^{27}$ Erst nach einigem Zögern akzeptierte Goethe die Campagne in Frankreich als eine zum Druck zu befördernde „Epoche meines Lebens“ und damit als Teil seiner Autobiographie. ${ }^{28}$

Der Eintrag vom 4. Oktober beginnt mit einer Szene, in der sich die besiegten deutschen Truppen in Sivry-les-Buzancy, einem kleinen Ort in den Ardennen, mit Gemüse in französischen Gärten versorgen:

\begin{abstract}
„Wir schlugen unser Lager unmittelbar bei Sivry, in dessen Umgegend wir noch nicht alles verzehrt fanden. Der Soldat stürzte in die ersten Gärten und verdarb was andere hätten genießen können. Ich ermunterte unseren Koch und seine Leute zu einer strategischen Fouragierung, wir zogen ums ganze Dorf und fanden noch völlig unangetastete Gärten und eine reiche, unbestrittene Ernte. Hier war von Kohl und Zwiebeln, von Wurzeln und andern guten Vegetabilien die Fülle; wir nahmen deshalb nicht mehr als wir brauchten, mit Bescheidenheit und Schonung. Der Garten war nicht groß, aber sauber gehalten, und ehe wir zu dem Zaun wieder hinauskrochen, stellt' ich Betrachtungen an wie es zugehe, $\mathrm{da} ß$ in einem Hausgarten doch keine Spur von einer Türe ins anstoßende Gebäude zu erkennen sei.“ (C S. 458)
\end{abstract}

Das deutsche Kommando beschließt in der Gegend zu rasten und die Offiziere werden für eine Nacht in Sivry einquartiert. Nun liegt gewissermaßen die spiegelverkehrte Situation zur französischen Einquartierung im Frankfurter Elternhaus vor: Dort hatte man den französischen Königsleutnant im Haus und nun ist Goethe selbst ein hochrangig Einquartierter in einem französischen Haus (allerdings nur für eine Nacht, während, wie dargestellt, der französische Generalgouverneur annähernd zwei Jahre lang in Goethes Elternhaus einquartiert war). Einquartierung hat, selbst wenn sie einvernehmlich erfolgt, immer mit Gewalt und Macht zu tun. Die Einquartierten besetzen einen Raum, den andere, meist dem politischen Gegner Zugehörige, ${ }^{29}$ als den eigenen betrachten. Zwischen den Parteien besteht indessen ein wechselseitiges Abhängigkeitsverhältnis, man muss sich doch aus jeweiligem Eigeninteresse miteinan- 
der arrangieren. Insofern ist mit der Situation der Einquartierung die Notwendigkeit der Selbstrelativierung bzw. der sich selbst relativierenden Wahrnehmung verbunden, die in der oben zitierten Stelle in Goethes Vermerk des schonenden Umgangs mit den fremden vegetabilen Ressourcen zum Ausdruck kommt. Goethe beschreibt den französischen Haushalt mit gleichsam ethnologischem Interesse (so wie Thorane im Hause Goethe die Tapeten und das gesamte Frankfurter Hauswesen beobachtet haben mag...):

\begin{abstract}
„Man trat nicht unmittelbar von der Straße in das Haus, sondern fand sich erst in einem kleinen, offenen, viereckten Raum, wie die Türe selbst das Quadrat angab; von da gelangte man, durch die eigentliche Haustüre, in ein geräumiges, hohes, dem Familienleben bestimmtes Zimmer; es war mit Ziegelsteinen gepflastert, links, an der langen Wand, ein Feuerherd, unmittelbar an Mauer und Erde $[\ldots]$.

Indessen wir uns freundlich mit den guten Menschen besprachen, bemerkt' ich erst wie architektonisch klug Anrichte, Gossenstein, Topf- und Tellerbretter angebracht seien. Diese nahmen sämtlich den länglichen Raum ein, den jenes Viereck des offenen Vorhauses inwendig zur Seite ließ. Nett und alles der Ordnung gemäß war das Geräte zusammengestellt [...].“ (C S. 459)
\end{abstract}

Es ist offensichtlich, wie wohlgefällig Goethes Blick auf der häuslichen Ordnung ruht, die es, zumal in den ungeordneten Kriegszeiten, erlaubt, die Räumlichkeit und den Zweck der sich in ihr befindlichen Einrichtungsgegenstände auf einen Blick zu erfassen. Und tatsächlich erweist sich das Haus als eine reflexive Ordnungsstruktur im weitergehenden Sinne. Wie einst der Generalgouverneur Thorane im Goethe'schen Elternhaus und die Fouragierer im französischen Hausgarten achten Goethe und die Seinen penibel darauf, die Ordnung des Hauses so wenig wie möglich zu stören und dem Hausherrn und seiner Familie respektvoll zu begegnen. Ja, man hilft ihnen sogar, sich gegen die einfachen, sich weniger geordnet verhaltenden deutschen Soldaten zu schützen (vgl. C S. 461). Und als die deutschen ,Gäste" beispielsweise im Nachbarhaus ein gemästetes Schwein requirieren und ihnen die Hausleute, deren Sympathien eher bei den deutschen Offizieren als bei ihren Nachbarn zu liegen scheinen, bei der Schlachtung des Schweins behilflich sind, wird einmal mehr deutlich, dass über die Grenzmarkierungen des Hauses Verhältnisse zwischen Innen und Außen, Deutschen und Franzosen, Fremden und Eigenen, Höherund Niedriggestellten reguliert werden, die eindeutige, d.h. binäre Konfronta- 
tionen außer Kraft setzen. In dem Maße, in dem Goethe die Franzosen als sozial und politisch differenziert zeichnet, werden über die Ordnungsfigur des Hauses Außen- und Binnendifferenzierungen einer reflexiven autobiographischen Selbstverortung ins Werk gesetzt, die den auf klarer und unversöhnlicher Grenzziehung beharrenden väterlichen Eigen- und Starrsinn längst hinter sich gelassen hat.

Von besonderer Signifikanz ist indessen der Schlussabschnitt der Einquartierungsszene, die kompositorisch auf den Eingang des Eintrags vom 4. Oktober zurückverweist. Goethe blickt sich nochmals in dem Haus um, in dem er zu Gast war:

\begin{abstract}
„Als ich mich in dem innern Zimmer umsah fand ich zuletzt eine Türe verriegelt, die ihrer Stellung nach in einen Garten gehen mußte. Durch ein kleines Fenster an der Seite konnt' ich bemerken daß ich nicht irre geschlossen hatte; der Garten lag etwas höher als das Haus, und ich erkannt' ihn ganz deutlich für denselben wo wir uns früh mit Küchenwaren versehen hatten. Die Türe war verrammelt und von außen so geschickt verschüttet und bedeckt, daß ich nun wohl begriff, warum ich sie heute früh vergebens gesucht hatte. Und so stand es in den Sternen geschrieben, daß wir, ohngeachtet aller Vorsicht, doch in das Haus gelangen sollten.“ (C S. 464)
\end{abstract}

Nicht nur betont der astrologische Hinweis wie bereits am Anfang von Dichtung und Wahrheit ${ }^{30}$ die autobiographische Bedeutsamkeit der Szene, auch nimmt die Absperrung zwischen Haus und Garten, die der französische Hausherr wohl vorgenommen hatte, um das Haus vor den deutschen Eindringlingen zu schützen, die Konfiguration des Elternhauses auf, wo der junge Goethe nur aus dem zweiten Stock in die ihm verschlossenen Gärten der Nachbarn blicken kann. Während man üblicherweise vom Haus in den zugehörigen Garten treten und in dieses zurückkehren kann, zeichnet die deutlich markierte Grenze von Haus und Garten sowohl in Dichtung und Wahrheit als auch in der Campagne in Frankreich eine reflexive Konfiguration, die an der Schwelle, an der versteckten und verrammelten Tür oder am Fenster, innehalten lässt. Das Ich ist an diesen Grenzmarkierungen auf sich selbst zurückgeworfen und kann, indem es sich diesseits und jenseits denkt (am Vortag war es im Garten, jetzt ist es im zugehörigen Haus), die getrennten Räume und damit auch die Episoden seines Lebens in Verbindung bringen. ${ }^{31} \mathrm{Im}$ Akt der autobiographischen Reflexion wird retrospektiv aufeinander bezogen, was im Erleben nicht oder noch nicht 
zusammenkommt. Bemerkenswerterweise handelt es sich beide Male um verschlossene „Paradiese“: Die Nachbargärten, die dem Kind Goethe in Dichtung und Wahrheit nicht zugänglich sind, erscheinen diesem als paradiesisch, ebenso wie die üppigen Gärten in Sivry-les-Buzancy, in denen Goethe trotz der Kriegslage Kohl, Zwiebeln und Karotten in Hülle und Fülle einheimsen kann, kontrafaktisch Paradiescharakter haben - insbesondere im Rückblick, als der Autobiograph nur noch hinaus- und zurückschauen kann, wo er war und nicht mehr hingelangt. In der Topik der Autobiographie erscheint das Paradies als jener verlorene Ort (und Raum - ein raumloser Raum jenseits aller Räumlichkeit), welcher die räumliche Figuration des auf den Lebensweg geworfenen Menschen motiviert. Das Elternhaus ist nur ein ambivalentes Substitut, das seinerseits im autobiographischen Rückblick als Kindheitsparadies erscheint, andererseits aber immer schon von jenen sozialen Privationen und Machtauftritten geprägt ist, die letztlich zur Vertreibung des autobiographischen Subjekts aus demselben führen. ${ }^{32}$

Die autobiographische Valenz verschlossener Paradiesgärten und nicht mehr zugänglicher Elternhäuser wird Goethe auf dem Rückzug in Trier am 29. Oktober 1792 bewusst. Speyer ist bereits von den Franzosen besetzt, die Übergabe von Mainz wird vorbereitet.

\footnotetext{
„Nichts fand man wahrscheinlicher und natürlicher als daß auch schon Coblenz von den Franken besetzt sei, und wie sollten wir unsern Rückweg antreten! Frankfurt gab man in Gedanken gleichfalls auf; Hanau und Aschaffenburg an einer, Cassel an der andern Seite sah man bedroht und was nicht alles zu fürchten!

$[\ldots]$

Mitten in diesem Unheil und Tumulte fand mich ein verspäteter Brief meiner Mutter, ein Blatt das an jugendlich-ruhige städtisch-häusliche Verhältnisse gar wundersam erinnerte. Mein Oheim Schöff Textor war gestorben, dessen nahe Verwandtschaft mich von der ehrenhaft wirksamen Stelle eines Frankfurter Ratsherrn bei seinen Lebzeiten ausschloß, worauf man, herkömmlich löblicher Sitte gemäß, meiner sogleich gedachte, der ich unter den Frankfurter Graduierten ziemlich weit vorgerückt war.“ (C S. 493f.)
}

Goethes Mutter fragt also im Auftrag des Frankfurter Rats an, ob ihr Sohn, Johann Wolfgang, bereit wäre, sollte er gewählt werden, die Stelle eines Frankfurter Ratsherren anzutreten, d.h. nach Frankfurt und wohl auch ins Elternhaus 
zurückzukehren. Damit ist Goethe unversehens an einen entscheidenden Wendepunkt bzw. an den Punkt einer lebenswichtigen Entscheidung gelangt. Entsprechend betroffen reagiert er:

„Vielleicht konnte eine solche Anfrage in keinem seltsamern Augenblicke anlangen als in dem gegenwärtigen; ich war betroffen, in mich selbst zurück gewiesen, tausend Bilder stiegen vor mir auf und ließen mich nicht zu Gedanken kommen. Wie aber ein Kranker oder Gefangener sich wohl im Augenblicke an einem erzählten Märchen zerstreut, so war auch ich in andere Sphären und Jahre versetzt." (C S. 494)

In der Erinnerung befindet sich das autobiographische Ich plötzlich wieder im paradiesischen Garten des Großvaters:

„Ich befand mich in meines Großvaters Garten, wo die reich mit Pfirsichen gesegneten Spaliere des Enkels Appetit gar lüstern ansprachen und nur die angedrohte Verweisung aus diesem Paradiese, nur die Hoffnung die reifste rotbäckigste Frucht aus des wohltätigen Ahnherrn eigner Hand zu erhalten, solche Begierde bis zum endlichen Termin einigermaßen beschwichtigen konnte." (C S. 494f.)

Schon im retrospektiv erinnerten kindlichen Paradies droht also der Hinauswurf. Goethe vergegenwärtigt sich in dem für ihn biographisch entscheidenden Moment die Laufbahn des Großvaters und ruft sich - als mögliches Spiegelbild seines eigenen künftigen Lebens - das Bild des nun verstorbenen Oheims vor Augen:

„Bei meiner letzten Durchreise durch Frankfurth hatte ich meinen Oheim im Besitz des Hauses, Hofes und Gartens gefunden, der als wackrer Sohn, dem Vater gleich, die höheren Stufen freistädtischer Verfassung erstieg. Hier im traulichen Familienkreis, in dem unveränderten alt bekannten Lokal, riefen sich jene Knaben-Erinnerungen lebhaft hervor und traten mir nun neukräftig vor die Augen.“ (C S. 495)

Goethe rekapituliert hier eine gestufte Erinnerung: In Anbetracht des mütterlichen Briefes erinnert er sich an seinen letzten Besuch in Frankfurt im Haus des Großvaters und Onkels. Letzterer wird als Hausherr, Besitzer von Haus, Hof 
und Garten, erinnert; das Haus selbst (,,in dem unveränderten alt bekannten Lokal") wird zum Ort der Erinnerung, so jedenfalls erinnert sich der Trierer Goethe, an die paradiesische Knabenzeit im großväterlichen Garten. Die Karriere eines reichsstädtischen Bürgers verbindet sich in der jugendlichen Vorstellung, wie Goethe anfügt, mit dem „süße[n] Gedanke[n]“ „,in der Brust eines jeden Republikaners", „an irgend einem Regimente Teil zu nehmen“ (C S. 495). Dies vergegenwärtigt sich jener Goethe, der gerade im Dienst eines Fürsten gegen das republikanische Frankreich von einem verlorenen Feldzug zurückkehrt! Daher muss er sich diese Knabenträume rasch verbieten:

\begin{abstract}
„Diesen freundlichen Kinderträumen konnt' ich mich jedoch nicht lange hingeben, nur allzuschnell aufgeschreckt besah ich mir die ahnungsvolle Lokalität die mich umfaßte, die traurigen Umgebungen die mich beengten und zugleich die Aussicht nach der Vaterstadt getrübt ja verfinstert. Mainz in französischen Händen, Frankfurt bedroht, wo nicht schon eingenommen, der Weg dort hin versperrt und innerhalb jener Mauern, Straßen, Plätze, Wohnungen, Jugendfreunde, Blutsverwandte vielleicht schon von demselben Unglück ergriffen daran ich Longwy und Verdun so grausam hatte leiden sehen; wer hätte gewagt sich in solchen Zustand zu stürzen!“ (C S. 495f.)
\end{abstract}

Die Heimat ist also (nicht zuletzt imaginär) besetzt, daher „der Weg dort hin versperrt". Dass es sich möglicherweise um ein vorgeschobenes Argument handelt und Goethe einfach keine Lust hatte, nach Frankfurt zurückzukehren, legt die sich anschließende Ausführung nahe, in der Goethe (sich selbst gegenüber) zu bedenken gibt, dass er auch unter anderen politischen Umständen das Angebot hätte ausschlagen müssen: Seit zwölf Jahren genießt er die Gunst des Weimarer Herzogs, dem er, Goethe, dient und der ihm die „Gelegenheit“" gegeben hat, „[s]ich zu entwickeln“ (C S. 496). Goethe bringt hier den Grundgedanken seines autobiographischen Konzepts in Anschlag, wie er es in der Vorrede zu Dichtung und Wahrheit formuliert hat. ${ }^{33}$ Carl August hat ihm gewissermaßen seine (Auto)Biographie ermöglicht - und nicht nur das: Goethe hat inzwischen zu anderer Häuslichkeit in einem anderen Staatsgebäude gefunden:

„[...] meine Dankbarkeit war ohne Grenzen so wie die Anhänglichkeit an die hohen Frauen Gemahlin und Mutter, an die heranwachsende Familie, an ein Land, dem ich doch auch manches geleistet hatte. Und mußte ich nicht zugleich 
jenes Zirkels neuerworbener höchstgebildeter Freunde gedenken, auch so manches andern häuslich Lieben und Guten was sich aus meinen treubeharrlichen Zuständen entwickelt hatte. Diese bei solcher Gelegenheit abermals erregten Bilder und Gefühle erheiterten mich auf einmal in dem betrübtesten Augenblick: denn man ist schon halb gerettet wenn man, aus traurigster Lage im fremden Land, einen hoffnungsvollen Blick in die gesicherte Heimat zu tun aufgeregt wird; so genießen wir diesseits auf Erden was uns jenseits der Sphären zugesagt ist." (C S. 496)

Der Gedanke an die besetzte Heimat hat die Gedanken von Frankfurt auf Weimar umgelenkt. Anstatt die Heimat im erinnernden Rückblick an die frühe Kindheit zu suchen, blickt Goethe aus der bedrängten Gegenwart nach vorne auf die Wahlheimat des Weimarer Fürstentums. Überhöht wird dieser Gedanke durch ein gleichsam heilsgeschichtliches, gleichwohl kaum christlich indiziertes Diesseits/Jenseits-Schema, das mit der Sphären-Metapher die autobiographische Topik der Himmelskörper-Konstellationen einzuholen scheint. Allerdings scheint sich Goethe selbst nicht ganz zu glauben und äußert den Verdacht, dass sich ,diese Beweggründe zunächst auf [s]ein Gefühl, auf persönliches Behagen, individuellen Vorteil zu beziehen schienen“ (C S. 496). Daher schiebt er weitere Argumente nach: Seine langjährige Tätigkeit (im Fürstendienst) hat ihn für die ,städtischen Bedürfnisse[] und Zwecke“ (C S. 497) ungeeignet gemacht. Ja, die Frankfurter Verfassung sieht vor, dass „eigentlich nur Bürger in den Rat aufgenommen werden sollten", und er selbst ist den Frankfurter Zuständen „,so entfremdet“, dass er sich nurmehr „,öllig als einen Auswärtigen" (C S. 497) betrachten kann.

Die angestellten Lektüren zeigen, dass der Auftritt und die Selbstwahrnehmung des autobiographischen Ichs räumlicher Strukturen und architekturaler Ordnungen bedarf, die seine Spiel- und Denkräume abstecken. Gerade die Häuser in Goethes autobiographischen Texten ${ }^{34}$ sind reflexive Konfigurationen und immer auch Bühnen politischer und sozialer Wechselbeziehungen, in denen sich das autobiographische Ich einerseits verorten und deren Grenzmarkierungen es andererseits hinter sich lassen kann. In diesem Sinn ist mit „Goethes Einquartierungen“ eine über den Kriegs- und Besatzungskontext hinausgehende Bedeutung autobiographischer Selbstdimensionierung gemeint, sind die autobiographischen Erfahrungsräume doch immer ,besetzt ${ }^{\star}$ - nicht zuletzt durch das autobiographische Ich und seine Rationalisierungsversuche. 


\section{Anmerkungen}

1 Vgl. Dilthey, 1981, S. 238: „Wenn wir auf die Vergangenheit zurückblicken, verhalten wir uns passiv; sie ist das Unabänderliche; vergebens rüttelt der durch sie bestimmte Mensch an ihr in Träumen, wie es anders könnte geworden sein. Verhalten wir uns zur Zukunft, dann finden wir uns aktiv, frei. Hier entspringt neben der Kategorie der Wirklichkeit, die uns an der Gegenwart aufgeht, die der Möglichkeit. Wir fühlen uns im Besitz unendlicher Möglichkeiten. So bestimmt dies Erlebnis der Zeit nach allen Richtungen den Gehalt unseres Lebens." Vgl. zum hermeneutischen Autobiographieverständnis WAGNER-EGELHAAF, 2005, S. 20-27.

2 LEJEUNE, 1994, S. 14.

3 Wolf, 1979, S. 176.

4 Vgl. grundlegend WeIGEL, 2002; BÖHME (Hg.), 2005; DüNNE/GÜNZEL (Hg.), 2006; BACHMANN-MEDICK, 2007.

5 Vgl. BÄHR u.a. (Hg.), 2007 sowie das von der DFG von 2009 bis 2012 an der Universität Münster geförderte Projekt „Topographien der Autobiographie“; http://www.uni-muenster.de/Germanistik/Lehrende/wagneregelhaaf_m/abgeschlossene_projekte/autobiographie_topographien.html; 06.03.2013. Stephan Berghaus, Kerstin Wilhelms und Philipp Pabst sei an dieser Stelle herzlich für ihre engagierte Mitarbeit im Projekt gedankt. Während der ,topographical turn' Figuration und Funktion von Orten in den Blick nimmt, geht es dem ,spatial turn' um die dezidiert räumliche Dimension kultureller Erscheinungen. In der Forschungspraxis wird häufig kein Unterschied zwischen beiden Ansätzen gemacht, zwischen denen es in der Tat auch zahlreiche Überschneidungen gibt. Die hier vorgenommene Unterscheidung hat denn auch eher heuristischen Wert. Vgl. auch die folgenden Ausführungen zu ,Ort' und ,Raum', auf deren begrifflicher Differenzierung tatsächlich zu insistieren ist.

6 Vgl. KANT, 1983, S. 71: „Vermittelst des äußeren Sinnes (einer Eigenschaft unsres Gemüts) stellen wir uns Gegenstände als außer uns, und diese insgesamt im Raume vor. Darinnen ist ihre Gestalt, Größe und Verhältnis gegen einander bestimmt, oder bestimmbar. Der innere Sinn, vermittelst dessen das Gemüt sich selbst, oder seinen inneren Zustand anschauet, gibt zwar keine Anschauung von der Seele selbst, als einem Objekt; allein es ist doch eine bestimmte Form, unter der die Anschauung ihres innern Zustandes allein möglich ist, so, daß alles, was zu den innern Bestimmun- 
gen gehört, in Verhältnissen der Zeit vorgestellt wird." Bähr, Burschel und Jancke verweisen zu Recht auf die räumliche Konstruktion des westlichen Subjekts und die grundlegende räumliche Verfasstheit seiner Betrachterposition (vgl. BÄHR/BURSCHEL/JANCKE, 2007, S. 8f.). Zur räumlichen Selbstkonstitution des Subjekts um 1800 vgl. BEHRENS, 2007 und BEHRENS/STEIGERWALD, 2010.

7 Vgl. Genette, 2008, S. 200. Dasselbe gilt freilich auch für die „Konzepte als Apperzeptionen" der Hermeneutik. Für die literaturwissenschaftliche Raumanalyse bedeutet dies, dass der wahrgenommene Raum immer mit dem Raum der Darstellung, d.h. der Räumlichkeit des Textmediums, in Relation gebracht werden muss.

8 Vgl. DERRIDA, 1976, S. 13: „Différance als Temporisation, différance als Verräumlichung. Wie geht das zusammen?“

9 Zur Raumperspektive in der Autobiographieforschung vgl. auch WAGNEREgelhaAf, 2010, S. 194-196 und DiES., 2012.

10 Dies ist in der sich aus unterschiedlichen Fachdisziplinen speisenden Forschung zum Raum nicht immer der Fall. Daher kommt es in Analysen wie in theoretischen Einlassungen häufig zu Ungenauigkeiten, Inkongruenzen und Widersprüchlichkeiten, die den Eindruck einer schwer zu operationalisierenden Heterogenität des Forschungsfelds ,Raum` hervorrufen. So systematisch Bähr, Burschel und Jancke in der Einleitung des von ihnen herausgegebenen Bandes Räume des Selbst argumentieren, indem sie u.a. auf die Standortgebundenheit des Betrachtersubjekts sowie die Eurozentrik der Außen/Innen-Dichotomie bezüglich der Vorstellung des ,Selbst ${ }^{\text {‘ }}$ verweisen, am Ende bekennen sie sich - und dies ist für einen Sammelband auch wohl kaum anders handhabbar - doch zur „Konzeptvielfalt“ (BÄHR/BURSCHEL/JANCKE, 2007, S. 11), wie sie insgesamt die Raumdebatte kennzeichnet. Watson verweist auf die unterschiedliche Konnotierung der Begriffe ,Raum' im Deutschen und ,space“ im Englischen und nimmt wichtige Begriffsklärungen vor: ,[...] the connotations of Raum are quite different from those of the English word ,room' that is its cognate, or of the more general term ,space'; Raum is more sweeping, philosophical, and metaphorical" (WATSON, 2007, S. 13). Auch Watson unterstreicht die räumliche Konstitution des autobiographischen Erzählers: ,$[\ldots]$ the focus on both the location and the position of an autobiographical narrator has importantly reshaped thinking about autobiography. The concept of location emphasizes not just the geographic, but the natio- 
nal, ethnic or racial, and gendered, sexual, social and life-cycle coordinates at which a narrator situates her- or himself. [...] The concept of position, by contrast, implies the ideological stances - multiple and heteroglossic rather than single and unified, despite the narrator's values - adopted by a narrator toward both self and others. Both concepts are inescapably spatial in their stress on emplacement, the juncture from which an articulation issues" (ebd., S. 15f.).

11 Vgl. Anm. 5.

12 Vgl. QUint. inst. 11, 2; vgl. WAGNER-EGELHAAF, 2010, S. 193.

13 Vgl. Augustinus, 1982, S. 254.

14 Vgl. HebeKus, 1995, S. 85.

15 Vgl. GoldmanN, 1994, S. 660-675.

16 Aus phänomenologischer Perspektive betont Gaston Bachelard die existenzielle Rolle des Hauses für den Menschen (BACHELARD, 1960, S. 35-69 und S. 70-103). Zu Marie Luise Kaschnitz' Erzählung Haus der Kindheit vgl. WAGNER-EGELHAAF, 2001.

17 Goethe, 1986, S. 15 (Nachweise im Folgenden im fortlaufenden Text unter der Sigle DW).

18 Vgl. Müller in Goethe, 1986, S. 1075 (Stellenkommentar zu Dichtung und Wahrheit, S. 1074-1298). Der Sternentopos findet sich noch im Titel des dritten Bandes von Emine Sevgi Özdamars autofiktionaler Trilogie Sonne auf halbem Weg (2006): Seltsame Sterne starren zur Erde (2003). Aus dem vormodernen Glauben, dass das Schicksal des Menschen in den Sternen vorbestimmt sei, ist ein wirkmächtiger autobiographischer Topos geworden, der die Repräsentation und Inblicknahme eines Lebens an eine momentane Konstellation bindet, aber dabei ihrer Veränderlichkeit und damit vielleicht auch der Flüchtigkeit des Lebens eingedenk bleibt.

19 Augustinus, 1982, S. 35f.: Augustinus spricht davon, dass er sich an seine frühen Kindertage nicht mehr selbst erinnern kann, sondern sich nur auf die Erzählungen anderer beziehen kann. Zur Problematik der Erinnerung bei Augustinus vgl. ebd., S. 254 und 256; vgl. WAGNER-EGELHAAF, 2005, S. $116 f$.

20 Freud, 1969, S. 258 beschreibt die Episode als ,Deckerinnerung' und das lustvolle Hinauswerfen des Geschirrs aus dem Haus als Wunsch, unliebsame Geschwister zu beseitigen, die als Konkurrenten in der mütterlichen Gunst empfunden werden. 
21 ,Enge“ und ,Weite' stellen grundlegende Raumparameter der Selbsterfahrung in Goethes Italienischer Reise dar, die gleichfalls Teil seiner Autobiographie ist. Vgl. dazu WAGNER-EGELHAAF, 2013.

22 Vgl. ARISTOT. poet. 6 („Die Tragödie ist Nachahmung einer guten und in sich geschlossenen Handlung von bestimmter Größe, in anziehend geformter Sprache, wobei diese formenden Mittel in den einzelnen Abschnitten je verschieden angewandt werden - Nachahmung von Handelnden und nicht durch Bericht, die Jammer und Schaudern hervorruft und hierdurch eine Reinigung von derartigen Erregungszuständen bewirkt.", ARISTOTELES, 1982, S. 19).

23 Vgl. die Beschreibung auf der Website des Frankfurter Goethe-Hauses: „Die aufwendige Treppenanlage, die fast ein Drittel des Gebäudes einnimmt, drückt den Willen des Hausherrn zur Repräsentation aus. [...] Das schmiedeeiserne Geländer im ersten Stock trägt die Initialen von Goethes Eltern: JCG und CEG“ (http://www.goethehaus-frankfurt.de/goethehaus/ 1-etage/vorsaal; 30.04.2012).

24 FREUd, 1917, S. 7.

25 Müller in GoETHE, 1986, S. 1099 (Stellenkommentar, S. 1074-1298).

26 Vgl. GoETHE, 1994, S. 386/924 (Stellenkommentar, S. 923-1003) (Nachweise im Folgenden im fortlaufenden Text unter der Sigle C).

27 HONOLD, 2002, S. 37 spricht von Goethes „Selbsthistorisierung“.

28 Goethe, 1994, S. 912 (Brief an Cotta vom 24. 6. 1822). Eine ,Epoche“ ist ein ,[bedeutsamer] Zeitraum“ oder „-abschnitt“ (DUDEN, Bd. 5, 2001, S. 183), macht also die Zeit räumlich wahrnehmbar. Die Grundbedeutung

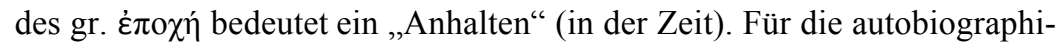
sche Bedeutung des Sternentopos (siehe Anm. 18) mag es nicht belanglos sein, dass ,Epoche“ in der Astronomie den „Zeitpunkt des Standortes eines Gestirns“"(DUdEN, Bd. 7, 2001, S. 277) bezeichnet.

29 Die Situation im revolutionären Frankreich ist differenzierter. Die Einquartierung bei möglicherweise politisch Gleichgesinnten, d.h. Revolutionsgegnern, ist gleichwohl mit einer Fremdwahrnehmung verbunden.

30 Vgl. Goethe, 1986, S. 15; siehe Anm. 28.

31 Diesen Hinweis verdanke ich Kerstin Wilhelms.

32 In diesem Zusammenhang verdiente Andreas Maiers autobiographischer Roman Das Haus eine einlässliche Untersuchung. Das Haus (2011) ist Teil zwei eines auf elf Bände angelegten autobiographischen Projekts mit dem Titel Ortsumgehung, dessen erster Band 2010 unter dem Titel Das 
Zimmer erschien. Die Lebensgeschichte ist dezidiert an Räumen orientiert, deren Kreise immer größer werden: Die Straße, Der Ort, Die Städte bilden weitere geplante Teile. Bislang ist so viel bekannt, dass Teil 10 Der Teufel und Teil 11 Der liebe Gott heißen sollen (vgl. http://www.dradio.de/dlf/ sendungen/buechermarkt/1326761/; 01.05.2012). Das elterliche Haus in Das Haus ist ein höchst ambivalenter paradiesischer Ort. Einerseits ist es Schutz- und Rückzugsraum eines ängstlichen Kindes, das sich vor der Außenwelt fürchtet, andererseits ist die bedrohliche Außenwelt längst im Inneren des Hauses ansässig, repräsentiert durch den Vater, der die gesellschaftlichen und menschlichen Machtstrukturen, die in der Welt draußen herrschen, längst und immer schon im Haus etabliert hat, so dass der Weg des kleinen Andreas letztlich auch zaghaft hinaus auf Die Straße führen muss. Gleichwohl bildet die architektonische Ordnung des Hauses ein Reflexionsmodell, in dem das autobiographische Ich sich seiner selbst bewusst werden kann: „Übrigens schien das Haus, kaum war ich allein, noch einmal um das Doppelte anzuwachsen, alle Räume erschienen mir plötzlich größer. Langsam lief ich die Treppe hinunter, immer wieder stehenbleibend und auf die Abwesenheit der Geräusche um mich herum lauschend. Die Atmosphäre eines jeden Raums nahm mich gefangen. [...] Alles war für sich und konnte so bleiben, vielleicht in Ewigkeit. Ganz langsam streunte ich durch die Räume. Ich ging ins Wohnzimmer, ins Eßzimmer, in die Küche ... aber warum ich da jeweils hinging, hätte ich nicht sagen können. Das Wohnzimmer war eigentlich eine Raumflucht aus zwei Zimmern, spärlich eingerichtet, die Decke wie im ganzen Haus nicht sonderlich hoch. [...] Eine unbewegte Welt. Vielleicht, wäre es immer so weitergegangen und wäre nie wieder jemand ins Haus zurückgekommen, hätte ich auch verhungern können im Haus, und es wäre mir trotzdem irgendwie richtig und als ein mir zugehöriger Teil meines eigentlichen Lebens vorgekommen. Vielleicht hätte ich beim Verhungern nicht einmal Hunger gespürt, sondern hätte nur in den Räumen dagestanden und später gelegen und wäre schließlich einfach gestorben, und der Tod hätte sich dann von dem Leben vorher gar nicht weiter unterschieden, und ich hätte es eigentlich auch gar nicht gemerkt.“ (MAIER, 2011, S. 161-164).

33 Vgl. GoEthe, 1986, S. 13f.: „Denn dieses scheint die Hauptaufgabe der Biographie zu sein, den Menschen in seinen Zeitverhältnissen darzustellen, und zu zeigen, in wiefern ihm das Ganze widerstrebt, in wiefern es ihn begünstigt, wie er sich eine Welt- und Menschenansicht daraus gebildet, 
und wie er sie, wenn er Künstler, Dichter, Schriftsteller ist, wieder nach außen abgespiegelt. Hierzu wird aber ein kaum Erreichbares gefordert, daß nämlich das Individuum sich und sein Jahrhundert kenne, sich, in wiefern es unter allen Umständen dasselbe geblieben, das Jahrhundert, als welches sowohl den willigen als unwilligen mit sich fortreißt, bestimmt und bildet, dergestalt daß man wohl sagen kann, ein Jeder, nur zehn Jahre früher oder später geboren, dürfte, was seine eigene Bildung und die Wirkung nach außen betrifft, ein ganz anderer geworden sein.“

34 Vgl. Berghaus, 2012.

\section{Literatur}

ARISTOTELES, Poetik, Griechisch/Deutsch, übers. und hg. von MANFRED FUHRMANN, Stuttgart 1982.

Augustinus, Aurelius, Bekenntnisse. Vollständige Ausgabe, eingel. und übertr. von Wilhelm Thimme, München 1982.

BACHELARD, Gaston, Poetik des Hauses, München 1960.

Bachmann-Medick, Doris, Spatial Turn, in: Dies., Cultural Turns. Neuorientierungen in den Kulturwissenschaften, 2. Aufl., Hamburg 2007, S. 284328.

Bähr, Andreas/Burschel, Peter/Jancke, Gabriele, Räume des Selbst. Eine Einleitung, in: BÄHR, ANDREAS u.a. (Hg.), 2007, S. 1-12.

BÄHR, ANDREAS u.a. (Hg.), Räume des Selbst. Selbstzeugnisforschung transkulturell, Köln u.a. 2007.

BEHRENS, RUDOLF, Räumliche Dimensionen imaginativer Subjektkonstitution um 1800 (Rousseau, Senancour, Chateaubriand), in: Räume der Romantik, hg. von INKA MÜLDER-BACH/GERHARD NEUMANN, Würzburg 2007, S. 27 63.

DeRS./STEIGERWALD, JÖRG: Raum - Subjekt - Imagination um 1800. Einleitende Überlegungen, in: Räume des Subjekts um 1800. Zur imaginativen Selbstverortung des Individuums zwischen Spätaufklärung und Romantik, hg. von DENS., Wiesbaden 2010, S. 1-13.

Berghaus, StePhan, Das topographische Ich. Zur räumlichen Dimension der Autobiographie in Goethes „Dichtung und Wahrheit“, Diss. Münster 2012.

BöHme, HaRtMut (Hg.), Topographien der Literatur. Deutsche Literatur im transnationalen Kontext. DFG-Symposion 2004, Stuttgart/Weimar 2005. 
Derrida, Jacques, Die différance, in: Ders., Randgänge der Philosophie, Frankfurt a. M. u.a. 1976, S. 6-37.

Dilthey, Wilhelm, Das Erleben und die Selbstbiographie, in: Der Aufbau der geschichtlichen Welt in den Geisteswissenschaften, Einleitung von MANFRED RIEDEL, Frankfurt a. M. 1981, S. 235-251.

DudEN, Bd. 5: Das Fremdwörterbuch, 7., neu bearbeitete und erweiterte Auflage, hg. von der Dudenredaktion, Mannheim u.a. 2001.

DudEN, Bd. 7: Das Herkunftswörterbuch. Etymologie der deutschen Sprache, 3., völlig neu bearbeitete und erweiterte Auflage, hg. von der Dudenredaktion, Mannheim u.a. 2001.

DÜNNE, JÖRG/GÜNZEL, STEPHAN (Hg.), Raumtheorie. Grundlagentexte aus Philosophie und Kulturwissenschaften, Frankfurt a. M. 2006.

Freud, Sigmund, Eine Schwierigkeit der Psychoanalyse, in: Imago. Zeitschrift für Anwendung der Psychoanalyse auf die Geisteswissenschaften 5 (1917), S. 1-7.

DERS., Eine Kindheitserinnerung aus „Dichtung und Wahrheit“, in: Studienausgabe, Bd. X: Bildende Kunst und Literatur, hg. von AleXANDER MitSCHERLICH u.a., Frankfurt a. M. 1969, S. 255-266.

GENETTE, GÉRARD, Strukturalismus und Literaturwissenschaft, in: Texte zur Literaturtheorie der Gegenwart, hg. und kommentiert von DOROTHEE KIMMICH u.a., Stuttgart 2008, S. 195-213.

Goethe, Johann Wolfgang, Aus meinem Leben. Dichtung und Wahrheit (Sämtliche Werke. Briefe, Tagebücher und Gespräche, I. Abt., Bd. 14), hg. von Klaus-Detlef Müller, Frankfurt a. M. 1986.

Ders., Campagne in Frankreich. Belagerung von Mainz (Sämtliche Werke, Briefe, Tagebücher und Gespräche, 1. Abt., Bd. 16), hg. von KlausDETLEF MÜLLER, Frankfurt a. M. 1994.

Goldmann, Stefan, Topos und Erinnerung. Rahmenbedingungen der Autobiographie, in: Der ganze Mensch. Anthropologie und Literatur im 18. Jahrhundert, hg. von HANS-JÜRGEN SCHINGS, Stuttgart/Weimar 1994, S. 660-675.

HebeKus, Uwe, Topik/Inventio, in: Einführung in die Literaturwissenschaft, hg. von Miltos Pechlivanos u.a., Stuttgart/Weimar 1995, S. 82-96.

HoNOLD, Alexander, Goethes Farbenkrieg, in: KulturPoetik 2, 1 (2002), S. 2443.

Kant, IMmanUel, Kritik der reinen Vernunft. Erster Teil, in: Werke in zehn Bänden, hg. von Wilhelm Weischedel, Bd. 3, Darmstadt 1983. 
LeJeune, PhiLIPPE, Der autobiographische Pakt, aus dem Französischen von WOLFRAM BAYER und DieTER HORNING, Frankfurt a. M. 1994.

MEIER, ANDREAS, Das Haus. Roman, Berlin 2011.

ÖZDAmar, Emine Sevgi, Sonne auf halbem Weg. Die Istanbul-BerlinTrilogie, Köln 2006 (bestehend aus: Das Leben ist eine Karawanserei hat zwei Türen aus einer kam ich rein aus der anderen ging ich raus [1992], Die Brücke vom Goldenen Horn [1998], Seltsame Sterne starren zur Erde [2003]).

Quintilianus, Marcus Fabius, Ausbildung des Redners. Zwölf Bücher, hg. und übers. von Helmut Rahn, 2 Bde., 3. Aufl., Darmstadt 1983.

Wagner-EgelhaAf, Martina, Autobiographie, 2. Aufl., Stuttgart/Weimar 2005 (Erstaufl. 2000).

DIES., Autobiographie - Rhetorik - Schrift. Zum Beispiel Marie Luise Kaschnitz, in: „Für eine aufmerksamere und nachdenklichere Welt“. Beiträge zu Marie Luise Kaschnitz, hg. von DIRK GöTTSCHE, Stuttgart/Weimar 2001, S. 8-26.

DIES., Zum Stand und zu den Perspektiven der Autobiographieforschung in der Literaturwissenschaft, in: BIOS. Zeitschrift für Biographieforschung, Oral History und Lebenslaufsanalysen 23, 2 (2010), S. 188-200.

DIES., ,Wir Cimmerier.' Goethe in Italien, in: Räumliche Dimension kultureller Konfrontationen, hg. von CARLA DAUVEN-KNIPPENBERG/ANNA SEIDEL, Amsterdam 2013 (im Druck).

WATSON, Julia, The Spaces of Autobiographical Narrative, in: BÄHR u.a. (Hg.), 2007, S. 13-25.

WeIgel, SigRID, Zum ,topographical turn'. Kartographie, Topographie und Raumkonzepte in den Kulturwissenschaften, in: KulturPoetik 2, 2 (2002), S. 151-165.

Wolf, Christa, Kindheitsmuster. Roman, Darmstadt/Neuwied 1979. 